\section{THE STERILIZATION OF WOOLEN BLANKETS AND UNIFORMS}

DUDLEY FULTON, M.D. (Los ANGeles)

Major, M. R. C., U. S. Army; Chief of Medical Service, Base Hospital AND

KENNETH J. STANIFORD, M.D. (FresNo, CALIF.)

Captain, M. R. C., U. S. Army; Chief of Laboratory Service, Base Hospital

CAMP LEWIS, AMERICAN LAKE, WASH.

The prevention and control of contagious diseases in Army cantonments and base hospitals is a complex problem which requires special measures to be worked out in sufficient detail to result, so far as possible, in automatic execution.

Contact infection, which includes hand-to-mouth infection and infection by the respiratory route, is accepted as being fundamentally important in the prophylaxis of contagions. A scheme, to be effective, should provide for measures involving early diagnosis, prevention of cross-infection in ambulances en route to the base hospital, rigid examination of patients in the receiving ward, the use of throat cultures to determine proper grouping of patients in wards, development of the cubicle system on the principle of isolation in each case, the wearing of face masks, and rigid ward discipline to develop and maintain the so-called aseptic technic. Important among preventive measures is proper sterilization of woolen blankets and uniforms.

The sterilizer issued the base hospital has proved satisfactory in the routine sterilization of cotton goods, surgical dressings and gowns, but destructive to woolen goods. It was found necessary, therefore, either to dispense with the routine sterilization of woolen goods or to modify the method used in sterilization of cotton goods, which consisted of sterilizing with from 12 to 15 pounds steam pressure (from 242 to $250 \mathrm{~F}$.) for from ten to thirty minutes.

Numerous experiments and modifications demonstrated that, in the sterilization of woolen goods, certain precautions must be observed, (1) to produce effective sterilization; (2) to avoid damage to woolen fibers, and (3) to prevent shrinkage. These precautions should be discussed.

\section{STERILIZATION}

Bacteriologic cultures of pneumococcus, streptococcus, staphylococcus, influenza bacillus, pyocyaneus and anthrax spores were effectively sterilized with 12 pounds of steam (242 F.) for ten minutes, or at atmospheric pressure $(212 \mathrm{~F}$.) for one hour, if the following precautions were taken:

1. That a vacuum of from 15 to 20 inches be established in the chamber of the sterilizer before the introduction of steam.

Experimental Proof.-Without initial vacuum, staphylococci and anthrax spores placed in the center of a bundle of blankets were not killed. With initial vacuum, all organisms were killed.

\section{That the sterilizer be not overfilled.}

Experimental Proof.-When the sterilizer was tightly filled, anthrax spores in the center of the sterilizer were not killed by 15 pounds of steam in fifteen minutes. When the woolen blankets and uniforms were loosely placed on trays of the sterilizer, all anthrax spores and other organisms were killed by 12 pounds pressure in ten minutes.

\section{DESTRUCTION OF WOOLEN GOODS}

The length of time woolen goods are exposed to heat is equally important to the degree of heat used (within the limits of 212 and $242 \mathrm{~F}$.)

Experimental Proof.-1. No damage to woolen goods resulted when subjected to steam at atmospheric pressure (212 F.) for one hour. 2. No damage resulted when woolen goods were subjected to 12 pounds pressure $(242 \mathrm{~F}$.) for ten or twelve minutes. 3. If exposed for a longer time, even at a lower temperature, slight or severe damage resulted, as shown by the following test:

Blankets subjected to 6 pounds pressure $(230 \mathrm{~F}$.) for thirty minutes evinced abnormal dryness, increased friability and loss of vitality of fibers and texture.

\section{SHRINKAGE}

Sudden change of temperature is the cause of shrinkage.

Experimental Proof.-1. Blankets and two olive drab coats, treated only once, shrank when, at the end of the sterilization, the sterilizer door was thrown open to allow rapid cooling. The blankets lost one-half inch in length and breadth and the olive drab coats lost from one-fourth to one-half inch in the collar. 2. A blanket was put through the sterilizing process eight times without shrinkage when attention was given to avoidance of sudden change of temperature by opening the sterilizer door only 4 inches for ten minutes.

\section{COMMENT}

The sterilizer issued to this base hospital was manufactured by the American Sterilizer Company and given the Serial No. 10482. As indicated above, blankets or uniforms should be placed loosely on the wire trays in the sterilizer. Fifty uniforms and 150 blankets, respectively, represent its maximum capacity in one sterilization. The capacity of the sterilizer is sufficient to meet the demands of this base hospital which has averaged from 1,000 to 2,100 patients during the past several months. Wrinkling of uniforms is avoided by the use of hangers. This reduces the capacity of the sterilizer to twenty-five uniforms.

For several months past, routine sterilization of all clothing except shoes, worn by patients with contagious diseases, has been accomplished before being sent to the receiving ward for storage. All blankets and bedclothing have likewise been sterilized immediately after a patient that has recovered from a contagious disease is discharged from the hospital or transferred to another ward. Early in our experience the atmospheric pressure method was preferred as it theoretically offered a greater margin of safety as to preservation of valuable property. The past five weeks, however, since time exposure has proved more important than the degree of heat used (within limits of 212 and $242 \mathrm{~F}$.), the shorter or pressure method has been adopted and found entirely satisfactory if conducted under reasonably careful observation.

\section{SUM MARY}

The following procedures effectively sterilize woolen goods without deterioration or shrinkage:

1. The woolen blankets or uniforms are placed on hangers or loosely on the trays in the sterilizer.

2. Sixty pounds of steam is introduced into the outer jacket of the sterilizer to prevent subsequent condensation of steam within the sterilizing chamber.

3. A vacuum of from 15 to 20 inches is created in the sterilizer chamber to facilitate penetration of the clothing by steam. 
4. Sterilization is performed with either (a) 0 pounds of steam for one hour, designated as the atmospheric pressure method, or (b) 12 pounds of steam for ten minutes, designated as the pressure method.

5. Again a vacuum of from 15 to 20 inches is produced to facilitate drying.

6. The door of the sterilizer is opened about 4 inches for ten minutes to allow gradual cooling of the contents of the sterilizer.

\section{Therapeutics}

\section{HEATSTROKE; INSOLATION}

The fact that the treatment of heatstroke is frequently unsatisfactory and the outcome frequently fatal is an excuse for repeatedly outlining our knowledge of this condition. Although sunstroke is perhaps more serious and more fatal than heat prostration, the latter not only may be fatal, but is even more likely to cause prolonged disability. With sunstroke the victim may become suddenly unconscious and die within a few hours or even minutes. He may not come out of the comatose condition, or he may die with evidence of heart weakness. He may have convulsions, and if he is not comatose, he may complain of dizziness, nausea, headache and disturbed vision; the pulse is rapid, and the temperature may be exceedingly high. In heat prostration the symptoms are those of shock, with weak pulse, subnormal temperature, clammy perspiration and heart failure. There may be nausea and vomiting.

\section{DIAGNOSIS}

A patient found comatose, and having been exposed to intense heat, must have his condition diagnosed from many other serious conditions that cause coma or convulsions. An epileptic convulsion, with coma, or several epileptic convulsions and a comatose condition, may be difficult to differentiate from heatstroke, in a patient unknown to the physician. The most important immediate distinction is the high temperature of the sunstroke as against a moderately increased temperature in the epileptic. The epileptic will be very cyanosed at first, but his color soon improves. A patient suffering from alcoholic stupor can generally be aroused, and the temperature is either normal or subnormal. The odor of alcohol on the breath does not preclude any other cause for the coma; in other words, the ambulance physician and the police surgeon should be very careful not to transport a stupid patient to jail because he smells of alcohol. Head injuries should always be sought for whenever a patient is found comatose. The temperature is likely to be subnormal. There may be symptoms of paralysis of one side or the other of the body. In coma from apoplexy or from injury to the head, with hemorrhage, there is stertorous breathing, with a temperature normal or a little above normal; the pupils are likely to be dilated, or one may be dilated even if the other is not; and the difference in the flexibility or mobility of the extremities will generally show, even in coma, that there is paralysis. Coma from uremic poisoning will show no great rise of temperature, if any, and there may be repeated convulsions. In diabetic coma the temperature will also be about normal; the breath may give the peculiar sweetish odor.
Even if the temperature is high and sunstroke is suspected, in every case the patient should be catheterized and the urine quickly examined for sugar and albumin.

In the coma of opium poisoning the pupils are small and pin-point, the breathing very slow, the pulse generally slow and full, and the temperature normal or subnormal. In chloral poisoning there is subnormal temperature, a weak pulse, the pupils may be slightly dilated, and the condition is that of collapse. These are only brief suggestions, to urge a more careful study of every person found comatose.

Gauss and Meyer ${ }^{1}$ studied 158 patients who suffered from heatstroke and were brought to the Cook County Hospital, Chicago. The seriousness of this affliction is shown by a death rate of 44.3 per cent. in these cases. It is interesting to note that Gauss and Meyer found that most of the patients gave a history of alcoholism, in greater or less degree. Most of the patients were thinly, that is, properly clothed for the summer season; most of the patients ate meat daily; a few ate meat three times a day. Some patients had prodromal symptoms of weariness for several days, or complained of headache and dizziness and great thirst, and some nausea and vomiting. Some patients had no prodrome whatever. When there was one or more days of more or less disturbing conditions or debility, the temperature was found not to be high; that is, these were more similar to heat prostration. When there was a very short period of symptoms of sunstroke, the temperature was generally, but not always found to be high. In some heat prostrations there was as low a temperature as 94, while in some cases of sunstroke the patients showed as high a temperature as 114 . The pulse rate was generally proportional to the temperature. Most of the comatose patients had involuntary bowel movements.

As emphasized above, the urine of all stupid patients should be examined. Gauss and Meyer found that of twenty-five patients whose urine was examined on the second day of illness, all showed hyaline and granular casts, and some showed albumin. Of the 158 patients, 129 were comatose when admitted, and fiftyeight died without regaining consciousness; ten had reiapses after gaining consciousness and died. Of those patients who recovered, the time of return to normal temperature varied up to three days.

It has long been noticed that a patient who has once had an attack of thermic fever readily has a recurrence with much less exposure to the sun. On the other hand, it has been found that a healthy white man can become acclimated to most intense tropical heat, and Shaklee $^{2}$ finds that the amount of sweating necessary to keep the temperature of the white man normal in a tropical climate is not excessive, even when he is doing physical work. He does find it essential, however, that the white man should have had for some little time a suitable diet, and should take up his work in the sun gradually, until he is able to do the full amount required.

Gradwohl and Schisler ${ }^{3}$ have studied thermic fever by laboratory methods, and find that insolation occurs when heat accumulates in the body and when the body does not excrete "its metabolic end-products, such as

1. Gauss, H., and Meyer, K. A.: Am. Jour. Med. Sc., 1917, 154, 554 .

2. Shaklec, A. O.: Philippine Jour. Sc. (B), 1917, 12, 1

3. Gradwobl, R. B. H., and Schisler, E.: Am. Jour. Med. Sc., 1917 154, 407. 